\title{
The chemical composition of $\beta$ Cephei and hybrid pulsators in the solar neighbourhood
}

\author{
Norbert Przybilla and Maria-Fernanda Nieva \\ Institut für Astro- und Teilchenphysik, Universität Innsbruck, \\ Technikerstr. 25/8, A-6020 Innsbruck, Austria \\ email: norbert.przybilla@uibk.ac.at, maria-fernanda.nieva@uibk.ac .at
}

\begin{abstract}
Cephei variables are the most prominent pulsators among the massive stars on the upper main sequence, extending into the class of the rare hybrid $\beta$ Cephei-SPB pulsators in the overlap region with the instability strip of the Slowly Pulsating B-stars. While the $\kappa$ mechanism has been identified as the driver of the pulsations, a comprehensive explanation of the excitation of the observed p- and g-modes is still lacking. In particular, the instability regions for these main-sequence B-type pulsators are still not fully consistent with any current opacity calculations. We have determined tight observational constraints on the chemical composition of a sample of $\beta$ Cephei and hybrid pulsators in the solar neighbourhood, covering all elements with abundances $\log (\mathrm{X} / \mathrm{H})+12>7.3$. The star sample turns out to be chemically homogeneous, with a metallicity $Z \approx 0.014$ and a non-solar abundance mix. The availability of accurate and precise abundances eliminates one of the two variables in the opacity calculations for asteroseismic applications, allowing to focus on (missing) atomic data.
\end{abstract}

Keywords. stars: abundances, stars: atmospheres, stars: early-type, stars: variables: other

\section{Introduction}

While asteroseismology sees widespread success in the study of the interiors of latetype stars its application to early-type stars is still challenging. $\beta$ Cephei variables of early B-type are the most prominent pulsators among the massive stars on the upper main sequence (with $M>8 M_{\odot}$ ), extending into the class of the rare hybrid $\beta$ Cephei-SPB pulsators in the overlap region with the instability strip of the Slowly Pulsating B-stars, which are of mid- and late-B type. The $\kappa$-mechanism driven by a metal opacity bump in the stellar envelope has been identified as the driver of the pulsations, see e.g. Aerts et al. (2010) for a discussion. However, a comprehensive explanation of the excitation of the observed p- and g-modes in the observed pulsators is still lacking. In particular, the instability regions for these main-sequence B-type pulsators are still not fully consistent with any current opacity calculations, see e.g. Salmon et al. (2012).

Opacity is the product of elemental abundances and atomic cross-sections, such that both input variables may contribute to problem in equal measure. While there is considerable improvement in the determination of the contribution of cross-sections (e.g. Bailey et al. 2015), little systematic and homogeneous work has been done on the determination of elemental abundances in $\beta$ Cephei stars. So far, the most comprehensive work on metallicity determinations for almost $50 \beta$ Cephei stars has been provided by Niemczura \& Daszyńska-Daszkiewicz (2005), from the analysis of low-resolution ultraviolet spectra. More detailed data on individual abundance in 9 objects were provided by Morel et al. (2006), analysing high-quality optical spectra on the basis of non-LTE line-formation.

Here, we want to discuss the impact of improvements on the abundance determinations recently achieved by Nieva \& Przybilla (2012, NP12), concentrating on the $\beta$ Cephei and hybrid pulsators included in their sample of early B-type stars in the solar neighbourhood. 


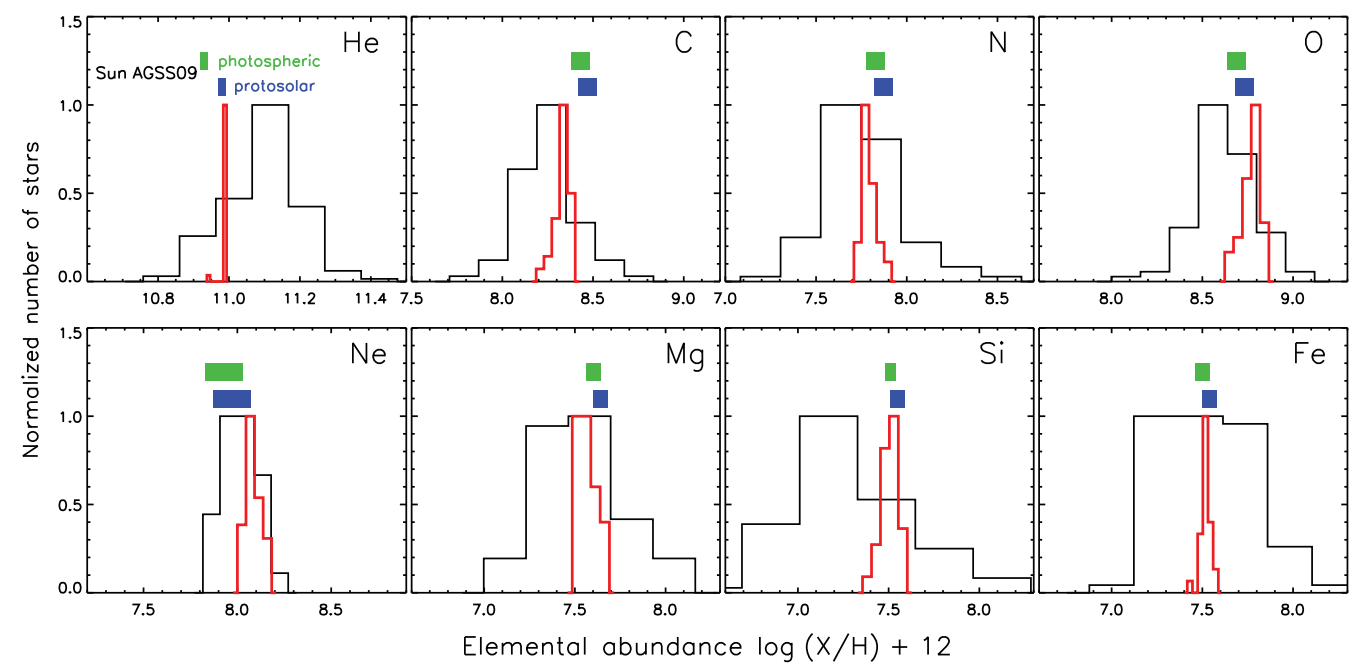

Figure 1. Abundance distributions for the astrophysically most relevant chemical species as derived from early B-type stars in the solar neighbourhood. Red histograms: data from NP12, establishing the cosmic abundance standard. Black histograms: literature data, see NP12 for details. Photospheric and protosolar abundances from AGSS09 are also indicated, the bars representing the range spanned by the $\pm 1 \sigma$-uncertainties.

\section{Observations and Analysis}

The combined star sample of NP12 and Nieva \& Simón-Díaz (2011, NSD11) contains in total 29 early B-type stars in the solar neighbourhood, at distances out to $\sim 400 \mathrm{pc}$. These include the four $\beta$ Cephei pulsators HD 61068 (PT Pup), HD 205021 ( $\beta$ Cep), HD 216916 (EN Lac) and HD 16582 ( $\delta$ Cet), as well as the hybrid $\beta$ Cephei-SPB pulsators HD 886 $(\gamma$ Peg) and HD 29248 ( $\nu$ Eri). For all the stars high-S/N $(S / N \approx 250-800)$, high-resolution spectra $(R=\lambda / \Delta \lambda \geqslant 40000)$ with wide wavelength coverage were available for analysis, taken with the Echelle spectrographs FOCES, FEROS, FIES and ELODIE, see NP12 and NSD11 for details.

Model calculations were carried out using a hybrid non-LTE approach as discussed in detail by Nieva \& Przybilla (2007, 2008) and Przybilla et al. (2011). In brief, hydrostatic, plane-parallel and line-blanketed LTE model atmospheres were computed with ATLAs9 (Kurucz 1993). Non-LTE line formation computations were then performed on these model structures using updated versions of the Detail and Surface codes (Giddings 1981; Butler \& Giddings 1985) and (updated) state-of-the-art non-LTE model atoms (He: Przybilla 2005; C II-IV: Nieva \& Przybilla 2006, 2008; N II: Przybilla \& Butler 2001; O I/II: Przybilla et al. 2000; Becker \& Butler 1988; Ne I/II: Morel \& Butler 2008; Mg II: Przybilla et al. 2001; Si III/IV: Becker \& Butler 1990; Fe II/III: Becker 1998; Morel et al. 2006).

Numerous spectroscopic indicators such as multiple ionization equilibria and Starkbroadened profiles of the Balmer and Paschen lines were used simultaneously to derive effective temperatures $T_{\text {eff }}$ and surface gravities $\log g$. Both, high accuracy and precision were achieved, with $1 \sigma$-uncertainties reduced to $\sim 1-2 \%$ in $T_{\text {eff }}$ and to $0.05-0.10$ dex in $\log g$. Absolute elemental abundances were constrained to $\sim 25 \%$ in systematic and to $\sim 10-20 \%$ in random $1 \sigma$-errors, a significant improvement over the factor $\sim 2$ in uncertainty typically found in previous work. The resulting synthetic spectra reproduce practically the entire observed optical/near-IR spectra of the target stars in very detail, with only a small percentage of spectral lines unaccounted for. 
Table 1. Individual elemental abundances.

\begin{tabular}{|c|c|c|c|c|c|}
\hline \multirow[t]{2}{*}{ Element } & Pulsators & \multirow{2}{*}{$\frac{\mathrm{CAS}}{\mathrm{NP} 12}$} & \multicolumn{2}{|c|}{ Sun - photospheric } & \multirow{2}{*}{$\begin{array}{l}\text { values } \\
\text { CLSFB11 }\end{array}$} \\
\hline & this work/NP12 & & GS98 & AGSS09 & \\
\hline $\mathrm{He}$ & $10.99 \pm 0.01$ & $10.99 \pm 0.01$ & & $10.93 \pm 0.01$ & \\
\hline $\mathrm{C}$ & $8.28 \pm 0.06$ & $8.33 \pm 0.04$ & $8.52 \pm 0.06$ & $8.43 \pm 0.05$ & $8.50 \pm 0.06$ \\
\hline $\mathrm{N}$ & $7.97 \pm 0.18$ & $7.79 \pm 0.04$ & $7.92 \pm 0.06$ & $7.83 \pm 0.05$ & $7.86 \pm 0.12$ \\
\hline $\mathrm{O}$ & $8.75 \pm 0.06$ & $8.76 \pm 0.05$ & $8.83 \pm 0.06$ & $8.69 \pm 0.05$ & $8.76 \pm 0.07$ \\
\hline $\mathrm{Ne}$ & $8.10 \pm 0.04$ & $8.09 \pm 0.05$ & $8.08 \pm 0.06$ & $7.93 \pm 0.10$ & . \\
\hline $\mathrm{Mg}$ & $7.56 \pm 0.03$ & $7.56 \pm 0.05$ & $7.58 \pm 0.05$ & $7.60 \pm 0.04$ & . \\
\hline $\mathrm{Si}$ & $7.49 \pm 0.06$ & $7.50 \pm 0.05$ & $7.55 \pm 0.05$ & $7.51 \pm 0.03$ & $\ldots$ \\
\hline $\mathrm{Fe}$ & $7.53 \pm 0.02$ & $7.52 \pm 0.03$ & $7.50 \pm 0.05$ & $7.50 \pm 0.04$ & $7.52 \pm 0.06$ \\
\hline
\end{tabular}

\section{Results}

One of the principal findings of NP12, supplemented by NSD11, is that contrary to all previous investigations the present-day chemical composition of the massive star population in the solar neighbourhood is highly homogeneous (see Fig. 1). Abundance fluctuations of less than $10 \%$ around the average were found, once the effects of a larger number of sources of systematic error, both on the observational (e.g. binarity, objects with circumstellar disks and chemically-peculiar stars) and modelling side (e.g. atomic data, stellar parameter determination) were minimised. The resulting set of early Bstar abundances constitutes the so-called cosmic abundance standard (CAS, NP12; see also Przybilla et al. 2008), establishing a second standard complementary to solar abundances, which have seen considerable revision recently (e.g. Grevesse \& Sauval 1998, GS98; Asplund et al. 2009, AGSS09; Caffau et al. 2011, CLSFB11).

The work of NP12 contains four $\beta$ Cephei pulsators and two hybrid $\beta$ Cep-SPB pulsators, for which we want to discuss in the following properties of this sample in context of the CAS. Table 1 summarises average abundances and the standard deviation for helium and all metals with abundances $\log (\mathrm{X} / \mathrm{H})+12>7.3$ in the pulsators. The elemental abundances in the pulsators are practically indistinguishable form the CAS, i.e. they are overall also chemically homogeneous. One exception is nitrogen, which shows a higher abundance on average (and a larger scatter) in the pulsators (see also Morel et al. 2006). The reason for this is that four out of the six pulsators are enriched in nitrogen, while N-rich stars were disregarded when establishing the CAS, as this is aimed at representing pristine abundances, unaffected by mixing with CN-cycled matter.

It would be worthwhile to investigate whether the opacity enhancement through enriched nitrogen can help to drive the observed pulsations. Note that while particle conversion in the $\mathrm{CN}$-cycle reduces carbon abundances by a mere $\sim 10 \%$, this corresponds to a change in nitrogen abundance by $\sim 60 \%$ on average in the present sample. However, note also that two of the pulsators show normal nitrogen abundances. As the $\beta$ Cephei and hybrid pulsators are stars evolved considerably off the zero-age main sequence, the larger fraction of stars showing $\mathrm{CN}$-cycled products on their surface may alternatively just be a consequence of mixing in the course of stellar evolution (e.g. Ekström et al. 2012; Maeder et al. 2014), unrelated to the driving mechanism of the pulsations.

Table 1 also shows that the CAS and the solar abundance standard are similar but not identical. Supported by arguments involving Galactic abundance gradients and Galactochemical evolution this was interpreted by NP12 as an indication that the Sun was not born near its present Galactocentric position, but has radially migrated from a birthplace around a Galactocentric distance of $\sim 5-6 \mathrm{kpc}$ to its current location over its lifetime. The Sun is therefore not representative for its current neighbourhood. Consequently, the abundance mix of the CAS should be preferred for opacity calculations for members of the young stellar population. Resulting mass fractions for hydrogen $X$, helium $Y$ and 
Table 2. Mass fractions for hydrogen, helium and metals.

\begin{tabular}{ccrrrr}
\hline \hline & \multirow{2}{*}{ CAS } & & \multicolumn{2}{c}{ Sun - photospheric values } \\
\cline { 2 - 2 } \cline { 5 - 6 } & NP12 & GS98 & AGSS09 & CLSFB11 \\
\hline$X$ & 0.710 & & 0.735 & 0.7381 & 0.7321 \\
$Y$ & 0.276 & & 0.248 & 0.2485 & 0.2526 \\
$Z$ & $0.014 \pm 0.002$ & & 0.017 & 0.0134 & 0.0153 \\
\hline
\end{tabular}

metals $Z$ are summarised for the CAS in Table 2, and compared to solar values. Note that the elements investigated by NP12 constitute the bulk of metal mass fraction. They have been supplemented by meteoritic solar values (AGSS09) for the less abundant metals and data from the Orion nebula for chlorine and argon from Esteban et al. (2004) as provisory CAS values. Note that these contribute little to the overall metallicity and any mismatch to true B-star abundances is well covered by the given uncertainties. The CAS metallicity of $Z \approx 0.014 \pm 0.002$ is compatible with modern values of the solar metallicity, but with a different abundance mix.

We conclude that accurate and precise abundances are now available for a small sample of pulsators, which should be representative also for other object at least out to distances of several hundred parsec from the Sun. The availability of these data eliminates one of the two variables in the opacity calculations for asteroseismic applications, allowing the focus to be put on (missing) atomic data.

\section{References}

Aerts, C., Christensen-Dalsgaard, J.,\& Kurtz, D. W. 2010,Asteroseismology (Dordrecht: Springer) Asplund, M., Grevesse, N., Sauval, A. J., \& Scott, P. 2009, ARAEA, 47, 481 (AGSS09)

Bailey, J. E., Nagayama, T., Loisel, G. P., et al. 2015, Nature, 517, 56

Becker, S. R. 1998, ASP Conf. Ser., 131, 137

Becker, S. R. \& Butler, K. 1988, A\& A, 201, 232

Becker, S. R. \& Butler, K. 1990, A\& A, 235, 326

Butler, K. \& Giddings, J. R. 1985, Newsl. Analysis of Astron. Spectra, No. 9 (Univ. London)

Caffau, E., Ludwig, H.-G., Steffen, M., et al. 2011, Sol. Phys., 268, 255 (CLSFB11)

Ekström, S., Georgy, C., Eggenberger, P., et al. 2012, A\& A, 537, A146

Esteban, C., Peimbert, M., García-Rojas, J., et al. 2004, MNRAS, 355, 229

Giddings, J. R. 1981, Ph.D. Thesis (Univ. London)

Grevesse, N. \& Sauval, A. J. 1998, Space Sci. Rev., 85, 161 (GS98)

Kurucz, R. L. 1993, CD-ROM No. 13 (Cambridge, Mass: SAO)

Maeder, A., Przybilla, N., Nieva, M. F., et al. 2014, A\&A, 565, A39

Morel, T., Butler, K., Aerts, C., Neiner, C., \& Briquet, M. 2006, A\&AA, 457, 651

Morel, T. \& Butler, K. 2008, A\& A, 487, 307

Niemczura, E. \& Daszyńska-Daszkiewicz, J. 2005, A\&A A, 433, 659

Nieva, M. F. \& Przybilla, N. 2006, ApJ (Letters), 639, L39

Nieva, M. F. \& Przybilla, N. 2007, A\&A, 467, 295

Nieva, M. F. \& Przybilla, N. 2008, A\&A, 481, 199

Nieva, M. F. \& Przybilla, N. 2012, A\&3A, 539, A143 (NP12)

Nieva, M. F. \& Simón-Díaz, S. 2011, A\& $A$, 532, A2 (NSD11)

Przybilla, N. 2005, A\&AA, 443, 293

Przybilla, N. \& Butler, K. 2001, A\&A, 379, 955

Przybilla, N., Butler, K., Becker, S. R., et al. 2000, A\&A, 359, 1085

Przybilla, N., Butler, K., Becker, S. R., \& Kudritzki, R. P. 2001, A\&A, 369, 1009

Przybilla, N., Nieva, M. F., \& Butler, K. 2008, ApJ (Letters), 688, L103

Przybilla, N., Nieva, M. F., \& Butler, K. 2011, J. Phys.: Conf. Ser., 328, 012015

Salmon, S., Montalbán, J., Morel, T., et al. 2012, MNRAS, 422, 3460 